\title{
ORIGINAL
}

ARTICLES

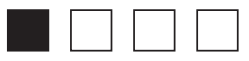

\section{Contraceptive Care for Women With Medical Conditions: A Qualitative Study to Identify Potential Best Practices for Primary Care Physicians}

Minji Michelle Kang, MPH; Timothy C. Guetterman, PhD, MA; Julie K. Prussack, MD; Allison Ursu, MD; Justine P. Wu, MD, MPH

BACKGROUND AND OBJECTIVES: Women with medical conditions are at higher risk of pregnancy-related morbidity and mortality than women without medical conditions. Thus, women who do not desire pregnancy should be offered contraceptive counseling and methods. The objective of this qualitative study was to identify potential best practices to guide primary care physicians (PCPs) in providing contraception and contraceptive counseling for women with medical conditions.

METHODS: We conducted semistructured qualitative interviews of 10 PCPs who routinely provide contraception including long-acting reversible contraception (LARC). To inform the data collection and analysis, we adapted constructs from the Theoretical Domains Framework. We coded transcripts and identified themes until saturation of our theoretical constructs was achieved.

RESULTS: Physician time constraints, lack of patient knowledge, and competing demands related to medical condition management were identified as barriers to contraceptive care. The study participants reported multiple strategies to mitigate these barriers. They emphasized providing reproductive health education in the context of an individual's chronic conditions and medications, educating about LARC methods, and using the US Medical Eligibility Criteria (US MEC) as a point-of-care clinical tool to guide contraceptive selection.

CONCLUSIONS: The study participants (PCPs experienced in contraceptive care), described multifaceted approaches to providing contraception for women with medical conditions, including tailored contraceptive education and use of the US MEC for clinical guidance. Future research is needed to assess if such strategies can improve patient outcomes and be adopted by PCPs who have less experience with contraceptive care.

(Fam Med. 2019;51(7):559-66.)

doi: 10.22454/FamMed.2019.499467

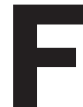
rom 2000 to 2014 , the US maternal mortality rate increased by $26 \% .^{1}$ Complications related to preexisting medical conditions, such as cardiovascular or renal disease, contribute to higher risks of severe morbidity ${ }^{2}$ and are estimated to account for one-third of maternal deaths. ${ }^{3}$ Contraceptive services are a critical strategy to prevent pregnancy-related morbidity and mortality related to preexisting medical conditions. $^{4}$

Primary care physicians (PCPs) are uniquely situated to address family planning within the context of preexisting conditions and related drug therapy. Observational and cross-sectional studies have shown that contraceptive counseling, particularly via a shared decision-making approach, has been associated with improved patient knowledge and decreased uncertainty about birth control options. ${ }^{5}$ However, there are insufficient data regarding how best to integrate contraceptive counseling and provision as a routine part of primary care for women with medical conditions. PCPs need strategies to assess pregnancy desires and provide tailored contraceptive care

From the University of Michigan, Michigan Medicine, Department of Family Medicine, Ann Arbor, MI. 
while balancing the competing demands of managing chronic disease and associated medications.

The primary aim of this qualitative study was to identify potential best practices for PCPs to provide contraception and contraceptive counseling for women with medical conditions. Thus, we purposefully sampled physicians who routinely manage chronic disease and offer full-spectrum reversible contraception, including long-acting reversible contraception (LARC). LARC methods refer to intrauterine devices and the subdermal implant, which are highly efficacious and do not contain estrogen. Therefore, LARC are important options for those with medical conditions that preclude estrogen use. $^{6}$

\section{Methods}

Participant eligibility was determined using criterion-i sampling technique, as described by Palinkas and colleagues, to select cases that meet predetermined criteria of importance. ${ }^{7}$ We sought study participants who were identified by peers as being committed to contraceptive care and thus could provide insight regarding the challenges and potential solutions to providing contraceptive care for women with medical conditions. The participants were eligible if they provide contraceptive counseling, prescriptions for contraceptive methods (eg, oral contraceptive pill, vaginal ring), and LARC procedures (insertion and removal) as part of their scope of practice. We used a snowball recruitment strategy, an appropriate technique to identify individuals who share similar characteristics, ${ }^{7}$ by approaching PCPs who met eligibility criterion and then in turn suggested other potential participants. Because we did not collect or report unique personal identifiers or personal health information, the University of Michigan Institutional Review Board deemed the study protocol exempt from review.

To inform our interview guide, data collection, and analysis, we selected constructs from the Theoretical Domains Framework (TDF), an integrated framework of constructs that reflect physician motivations, clinical behavior, and clinical behavior change. ${ }^{8}$ Based upon factors known to be associated with contraceptive provision by PCPs, we chose and adapted the following high-priority constructs ${ }^{9-11}$ : (1) knowledge (features of contraceptive methods, the impact of medical conditions on reproductive health and vice versa, application of general knowledge to specific clinical situations); (2) counseling and procedural skills (counseling tailored to women with medical conditions, LARC provision); (3) beliefs about capabilities (self-perception of ability to deliver contraceptive care to women with medical conditions, prior training in reproductive health/maternal health); (4) environmental context and resources (barriers and facilitators to providing contraceptive care for women with medical conditions).

The team consisted of author J.W. who is a family physician with expertise in family planning research, M.K. who is a maternal and child health public health researcher, and T.G. who is a qualitative methods expert. J.W. and T.G. conducted the interviews in a private setting in person or over the phone according to participant preference. We obtained verbal consent to conduct and audio-record the interview. No personal incentives were offered for participation. The interviews lasted 30 to 60 minutes and were transcribed verbatim.

During the interviews, participants answered questions regarding demographics and clinical training (gender, primary specialty, years in practice, percentage of patients who are reproductive-age women). Using an interview guide, J.W. and T.G. asked participants to describe their approaches to wellness exams and contraceptive counseling and then to consider how these approaches may be similar or different for women with medical conditions (eg, diabetes, hypertension).
JW and MK conducted the qualitative data analysis. The interview transcripts were uploaded and organized using MAXQDA software (VERBI GmbH, Berlin, and Germany Version 12.3.1). We conducted analysis using a template coding method described by Crabtree and Miller. ${ }^{12}$ First, our initial coding template was driven by the adapted TDF constructs. After independently reading through several transcripts, we combined some of the original codes and also generated in-vivo codes that captured findings not originally anticipated. We then discussed, compared, and reconciled differences in coding to create a consensus code template. This template included code names with clear operational definitions so that codes could be applied consistently. ${ }^{13}$ Finally, the codes were compared, contrasted, and synthesized to identify major themes and patterns. J.W. and M.K. recorded reflective memos during this process. Interviews continued until we achieved theoretical efficiency, defined as the point at which data are sufficient to address theoretical constructs. ${ }^{14}$ To challenge any biases or preconceptions that may have informed the final interpretation by J.W. and M.K., T.G. reviewed coding documents and reflective memos written by J.W. and M.K. We also conducted member checking ${ }^{15}$ with two of our participants (A.U., J.P.) who each independently read anonymized summaries of the qualitative codes and documented their reflections in memos and brief summaries. Their feedback was consistent with the team's key findings and provided insight into motivations behind study participants' desires to promote LARC use.

\section{Results}

The sample ( $\mathrm{n}=10)$ was comprised of PCPs who were all family physicians, predominantly female $(80 \%)$ and within 5 years posttraining $(80 \%)$. Half had completed fellowship training in maternal health or reproductive health and $40 \%$ currently provide obstetrics services 
(Table 1). Table 2 summarizes the process by which the adapted TDF constructs informed our qualitative coding and analysis. Our central themes relate to the study participants': (1) confidence in their contraceptive skills and knowledge; (2) identified barriers to contraception care; (3) counseling that emphasizes the potential impact of an individual's medical conditions and drug therapy on her reproductive health and vice versa; (4) routine discussion of LARC methods though with varied emphasis on LARC as a first-line option; and (5) use of the US Medical Eligibility Criteria (US MEC) as an evidence-based resource to guide contraceptive management for women with medical conditions. Each of these is elaborated below.
The study participants expressed confidence in their general contraceptive skills and their ability to tailor contraceptive counseling for women with medical conditions. Reflecting our sampling approach, the study participants described comprehensive understanding of different contraceptive methods and how to match methods to individual preferences. They were familiar with patient characteristics that would preclude the use of estrogencontaining methods (eg, poorly controlled diabetes, smoker over the age of 35 years), and emphasized the advantages of LARC methods in these situations as described by this participant: ...especially as someone who does long-acting reversible contraception, which, you know, are safe in most women who have complex medical conditions, I can tell women that these methods won't raise your blood pressure, not going to make diabetes worse, you can take them with all your other medications and they're going to provide you really good birth control until the time you want to become pregnant or until you need a new device placed. (P02)

The study participants identified lack of patient knowledge, time constraints, and competing demands related to managing chronic conditions as barriers to contraceptive care.

Table 1: Participant Demographics

\begin{tabular}{|c|c|}
\hline Characteristic & n (\%) \\
\hline \multicolumn{2}{|l|}{ Gender } \\
\hline Female & $8(80)$ \\
\hline Male & $2(20)$ \\
\hline \multicolumn{2}{|l|}{ Primary Specialty } \\
\hline Family medicine & $10(100)$ \\
\hline \multicolumn{2}{|l|}{ Clinical Setting } \\
\hline Academic center & 7 \\
\hline Multispecialty private practice & 2 \\
\hline Private practice/academic practice & 1 \\
\hline \multicolumn{2}{|c|}{ Fellowship Training in RH/MCH } \\
\hline Yes & $5(50)$ \\
\hline No & $5(50)$ \\
\hline \multicolumn{2}{|l|}{ Years in Practice } \\
\hline Mean & 4.6 \\
\hline Median & 3 \\
\hline \multicolumn{2}{|c|}{$\%$ of Patients Who Are Reproductive-Aged Women* } \\
\hline Mean & $43.9 \%$ \\
\hline Range & $20 \%-90 \%$ \\
\hline \multicolumn{2}{|c|}{ Currently Provide Obstetrics } \\
\hline Yes & $4(40)$ \\
\hline No & $6(60)$ \\
\hline \multicolumn{2}{|l|}{ LARC Provision } \\
\hline Yes & $10(100)$ \\
\hline
\end{tabular}

Abbreviations: $\mathrm{RH} / \mathrm{MCH}$, reproductive health/maternal and child health; LARC, long-acting reversible contraception (intrauterine devices and the subdermal implant).

* Represents 9 out of 10 participant responses. 
Table 2: Adapted Definitions of Constructs From the Theoretical Domains Framework* and Linkages to Key Findings/Themes

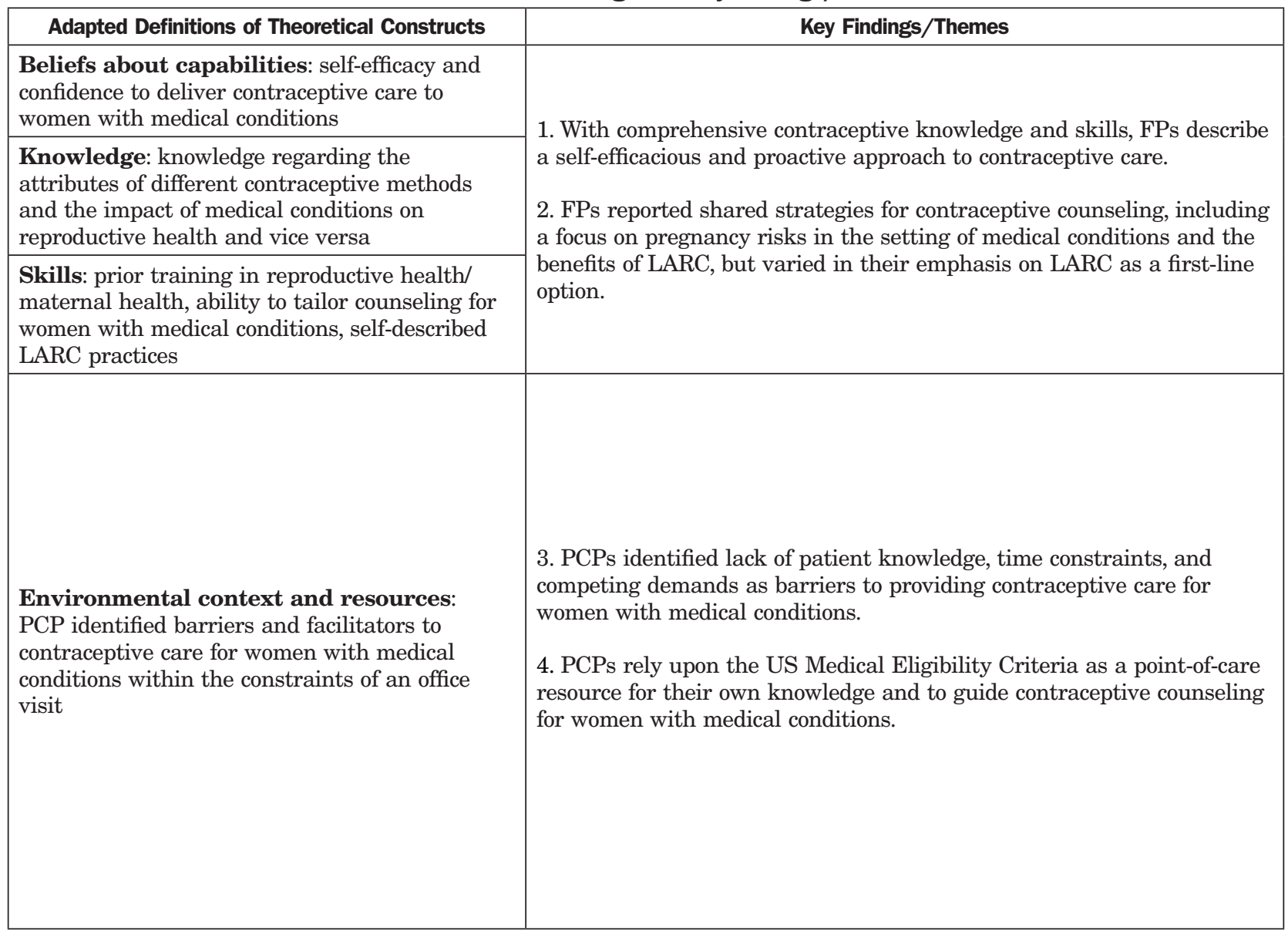

*Michie S, Johnston M, Abraham C, et al. Making psychological theory useful for implementing evidence based practice: a consensus approach. BMJ Quality \& Safety 2005; 14:26-33.

Study participants noted that many patients have inadequate understanding of the potential impact of their medical conditions on reproductive health outcomes and implications for birth control selection. They also expressed concerns about patients receiving inaccurate information from family, friends, and online forums that exaggerate the risks of LARC use:

..sometimes people don't even necessarily know they have a chronic condition or understand why that would change their [contraceptive] options...I think also word of mouth and talking to other women could be more hurtful in those situations, where some of their only options might be long acting reversible contraception, and if they've already heard bad stories about someone getting pregnant on an IUD, then it can make it even harder to provide them with the care...it's harder for them to learn about those things on the internet or by talking to people. (P08)

Study participants acknowledged that juggling multiple competing demands leaves less time for contraceptive discussions. This strain is particularly felt during wellness visits. One participant explained that additional visits may be necessary to address contraception:

...if they have diabetes we have to spend a good amount of time talking about that, and catching up on it and talking more about diet and exercise and weight loss and diabetes-related concerns. And we then probably have a lot less time to talk about contraception than someone who is otherwise healthy...maybe they'll have to come back to talk about it more. (P05)

Multiple strategies to overcome these barriers emerged from the analysis. Table 3 summarizes strategies to improve contraceptive counseling and provision and potential practice implications for all patients, and not just those with medical conditions. Strategies include: (1) eliminating unnecessary physical exam elements, including pelvic or breast exams; (2) offering bridge contraception until a patient can schedule a 
Table 3: General Strategies to Integrate Contraceptive Care During Clinical Visits

\begin{tabular}{|c|c|c|}
\hline Strategies & Illustrative Quote & Possible Practice Implications \\
\hline $\begin{array}{l}\text { Eliminate unnecessary } \\
\text { physical exam elements, } \\
\text { especially pelvic and/or } \\
\text { breast exams }\end{array}$ & $\begin{array}{l}\text {... I try to just do things for my } \\
\text { physicals that are more evidence- } \\
\text { based, so, my physical aspect of } \\
\text { it is very limited... (P04) } \\
\text {... If they're due for a pap I'll do } \\
\text { their pap on that visit. I don't do } \\
\text { bimanuals...unless they have a } \\
\text { complaint. ...the breast exam, } \\
\text { I tend to only do if they have a } \\
\text { complaint or any issues in the } \\
\text { past. (P09) }\end{array}$ & $\begin{array}{l}\text { Elimination of low-value exams that are not evidence- } \\
\text { based creates more time for "high-value" discussions, } \\
\text { including chronic care management, preconception care, } \\
\text { and contraceptive care. }\end{array}$ \\
\hline $\begin{array}{l}\text { Offer bridge birth control } \\
\text { method until LARC } \\
\text { insertion visit }\end{array}$ & $\begin{array}{l}\text { I don't typically insert a Mirena } \\
\text { on the same day as a well-wom- } \\
\text { an, unless they've been planning } \\
\text { that, but I would encourage that } \\
\text { if they're considering that, then } \\
\text { maybe getting a Depo today... } \\
\text { (P09) }\end{array}$ & $\begin{array}{l}\text { For physicians who cannot accommodate same-day } \\
\text { LARC insertion, they can offer same-day birth control as } \\
\text { a "bridge" to mitigate the risk of unintended pregnancy } \\
\text { until the day of LARC insertion. }\end{array}$ \\
\hline $\begin{array}{l}\text { Give contraceptive } \\
\text { information handouts } \\
\text { to patients who are not } \\
\text { ready to decide on a } \\
\text { method }\end{array}$ & $\begin{array}{l}\text { I think quick strategies, hand- } \\
\text { outs for patients to kind of plant } \\
\text { the seed that yes, we do birth } \\
\text { control here, so even if today } \\
\text { is not the day you want to talk } \\
\text { about it, let me give you some } \\
\text { good information so that next } \\
\text { time, or when you're ready, we } \\
\text { can have this conversation. (P02) }\end{array}$ & $\begin{array}{l}\text { Physicians should have educational resources readily } \\
\text { available to inform and prime the patient for future } \\
\text { contraceptive discussions. }\end{array}$ \\
\hline
\end{tabular}

Abbreviation: LARC, long-acting reversible contraception (intrauterine devices and the subdermal progestin implant).

LARC insertion visit; and (3) giving educational information to patients who may be interested but are not ready to decide about contraception.

Contraceptive care strategies that were specific to women with medical conditions are also identified and described in detail below.

\section{Study Participants Emphasized the Potential Impact of an Individual's Medical Conditions on Her Reproductive Health} (Fertility, Pregnancy, and Contraception) and Vice Versa For example, study participants felt strongly that it was their responsibility to educate about the implications of unplanned pregnancy related to specific conditions, particularly for those who have advanced disease (eg, poorly controlled diabetes).
Therefore, they offer a combination of preconception care, intensification of medical management, and/or contraception as appropriate. Furthermore, PCPs should inquire about pregnancy desires and offer contraception to women who use teratogenic medications (ie, may cause fetal defects):

...So when we talk about diabetes, we talk about...how does it affect your kidneys, and your heart, but also, how does it affect your ability to get pregnant, or, if you got pregnant what might be the outcome or your health, or how would your health be affected?... it's been a responsibility of primary care doctors to, when they're refilling a medicine that may be teratogenic, asking if someone is planning a pregnancy or even just mentioning that there are contraceptive methods that offer low to no hormones that would be very safe for them to use, would they be interested in learning more about that. (P02)

Study participants also felt it was important to raise awareness that some contraceptive methods may actually improve certain medical conditions and associated quality of life. For example, one participant described educating a patient that her migraines may be ameliorated with menstrual suppression:

...so if they're a patient with horrific migraines who gets it every time she has her period well then let's help her not have a period. And well let's think about that actually 
as a way to prevent her migraines, not just treating her with migraine medication. So if there's a way that the reproductive health condition, by addressing that, it'll improve the chronic illness, that's one way we can make it better. (P02)

Study Participants Routinely Discussed the Benefits of LARC, Although They Varied in Their Emphasis on LARC as a FirstLine Option

Given the superior contraceptive efficacy and safety of LARC methods, the study participants agreed that LARC methods are important options for women with medical conditions. Some felt strongly that LARC methods should be presented first and prioritized over short-acting methods (eg, pill, patch, shot) or coital-dependent methods (eg, condoms, withdrawal). One participant prioritized LARC methods first during counseling:

...I bring up LARC immediately, even if they say "I want the pill," I say "have you ever considered an IUD or an implant or a shot as an option? Because the difference is, you don't have to remember to take anything." (P03)

Others cautioned against a LARCfirst approach as being physiciancentric and advocated that patient preferences should guide contraceptive counseling:

...I think that LARC devices are wonderful, but they're not for everyone... for some that efficacy is not the most important thing to our patients even though it's something that we, as a provider community, often prefer. (P01)

...I don't think there's one ideal method of contraception, I think it is an individualized decision. We have to meet in the middle with the patient's thoughts about how they can best prevent pregnancy or use contraception for other reasons. (P07)

\section{Study Participants Relied Upon} the US Medical Eligibility

Criteria as a Point-of-Care

Clinical Tool to Guide

Contraceptive Selection for

Women With Medical Conditions

While the study participants reported using several contraceptive resources, they described the US Medical Eligibility Criteria (US MEC) as a highly valued point-ofcare clinical tool. Developed and updated regularly by the Centers for Disease Control and Prevention (CDC), the US MEC summarizes the relative risks and benefits of different birth control methods based upon patient characteristics (eg, age, smoker, parity) and the presence of medical conditions (eg, diabetes, seizures, HIV, morbid obesity). ${ }^{16}$ Although not all study participants could recall the exact name of the US MEC, they could describe its function and how to access the resource in multiple formats (phone app, summary chart). Some also found the US MEC useful to engage patients in contraceptive discussions:

...for most other, uncommon medical conditions, I'll make sure that there's, what both the CDC and the WHO have put together tables of which birth control is safe, which one you should use caution, and etcetera in patients with patients with various medical conditions. So if it's something more rare, I'll usually look things up on that table. I might even look through that with the patient and then we'll try to find something that works well for them. (P07)

\section{Discussion}

This qualitative study highlights the experiences of PCPs who routinely provide the full spectrum of reversible contraceptive methods, including LARC, to women with medical conditions and thus, serve as a potential source of best practices. Overall, this cohort was confident and comfortable with contraceptive counseling in the context of individual pregnancy desires, personal preferences, preexisting medical conditions, and drug therapy. Because all of the study participants were LARC providers as selected by eligibility criteria, it was not surprising that they emphasized the importance of LARC education. These findings underscore the importance of resident training in contraception, including LARC provision, which is associated with subsequent provision of these services in practice. ${ }^{17,18}$

While approximately half of the study participants in this study completed fellowship training in obstetrics or family planning, this additional training is neither necessary to provide contraception nor practical for the vast majority of clinicians. One possible strategy to train PCPs who have already completed residency is the expansion of regional centers that provide high volume procedural training over a short amount of time, and have been associated with significant improvements in physician knowledge and comfort with contraceptive counseling, although less robust results in increasing LARC provision. ${ }^{19}$

Study participants prioritized family planning during wellness visits despite time constraints; however, PCPs who do not routinely provide contraception or are not trained in LARC procedures may not feel as comfortable or motivated to do so. One possible strategy to encourage contraceptive discussions is to educate both physicians and patients about ways in which certain birth control methods may actually improve medical conditions (eg, treating chronic anemia by reducing menstrual loss) or at a minimum, not worsen preexisting conditions. Another possible approach is to raise PCP awareness about the need to provide contraceptive counseling 
when prescribing teratogenic medications, an intervention that has been associated with greater likelihood of contraceptive use. ${ }^{20}$

PCPs in this study agreed that LARC methods are well suited for women with medical conditions, particularly those with contraindications for estrogen or severe comorbidities that increase the risk of pregnancy-related complications. However, some were concerned about an overemphasis of a LARC-first counseling approach, that supports a growing concern among patients, clinicians, and experts that strong prioritization of LARC methods over others is a coercive counseling approach that undermines reproductive autonomy. ${ }^{21,21}$ Women with medical conditions that preclude the use of estrogen are often considered ideal candidates for LARC methods and may be particularly vulnerable to this type of directive contraceptive counseling. Prior studies support the use of a shared decision-making approach to contraceptive counseling, which prioritizes patient preferences and decision making via an interactive patient-physician discussion..$^{22}$

The study participants valued the US MEC as a point-of-access reference and even as a communication tool to facilitate patient counseling. Routinely updated by the Centers for Disease Control and Prevention (CDC), the US MEC is an evidencebased resource that summarizes the relative benefits and risks of different birth control methods in the context of over 60 medical conditions, as well as drug therapies, commonly encountered in primary care including hypertension, diabetes, migraine headaches, and use of antiretroviral and anti-epileptic medications. ${ }^{16}$ The US MEC recommendations are summarized in a color-coded chart that can be printed double-sided or accessed online. The US MEC can also be accessed via a smartphone app maintained by CDC. Despite its utility and availability in multiple formats, prior studies indicate that PCP awareness and implementation of the US MEC guideline remain suboptimal. ${ }^{11,23}$ Based on our study findings, widespread adoption of the US MEC in clinical practice may improve PCPs knowledge and confidence related to recommending contraception under various medical circumstances, which in turn could improve the quality of contraceptive counseling.

This study has limitations. Most of the study participants were based in academic centers and were within 5 years of residency training. Half had received training that would be expected to increase their familiarity with contraceptive counseling and procedures. The study participants' motivations, priorities, and experiences cannot be generalized to PCPs in community settings or PCPs who do not have as much experience with contraceptive care. However, the participants' experience with contraception care uniquely positions them to describe strategies and best practices. The study participants self-reported their clinical practices, which were not corroborated with chart review or patient interviews.

In summary, the study participants, PCPs who were experienced in contraceptive care, described multifaceted approaches to providing contraception and contraceptive counseling for women with medical conditions. The strategies included but were not limited to patient education tailored to medical history and preferences, routine discussion of LARC options, and reliance on the CDC US MEC as a clinical tool. Future research is needed to assess if such strategies can improve patient outcomes, and, if so, how to support and educate PCPs who may be less experienced with contraceptive care in adopting these practices.

FUNDING STATEMENT AND DISCLAIMER: Dr Wu receives support from the Eunice Kennedy Shriver National Institute of Child Health and Human Development (NICHD) under award number 1K23HD084744-01A1. The content is the responsibility solely of the authors and does not necessarily represent the official views of the NICHD. The funders had no role in the design and conduct of the study; collection, management, analysis and interpretation of the data; preparation, review, and approval of the manuscript; or decision to submit the manuscript for publication.

CORRESPONDING AUTHOR: Address correspondence to Dr Justine P. Wu, Assistant Professor, University of Michigan Department of Family Medicine, 1018 Fuller Street, Ann Arbor, MI 48104. 734-998-7120. Fax: 734-9987335. justinep@med.umich.edu.

\section{References}

1. MacDorman MF, Declercq E, Cabral H, Morton C. Recent Increases in the U.S. Maternal Mortality Rate: Disentangling Trends From Measurement Issues. Obstet Gynecol. 2016;128(3):447-455

2. Admon LK, Winkelman TNA, Zivin K, Terplan M, Mhyre JM, Dalton VK. Racial and ethnic disparities in the incidence of severe maternal morbidity in the United States, 2012-2015. Obstet Gynecol. 2018;132(5):1158-1166.

3. Centers for Disease Control and Prevention. Pregnancy mortality surveillance system. Trends in pregnancy-related deaths. 2017; https://www.cdc.gov/reproductivehealth/maternalinfanthealth/pmss.html. Accessed October 8, 2018.

4. Gavin L, Moskosky S, Carter M, et al; Centers for Disease Control and Prevention (CDC). Providing quality family planning services: Recommendations of CDC and the U.S. Office of Population Affairs. MMWR Recomm Rep. 2014;63(RR-04):1-54.

5. Perritt JB, Burke A, Jamshidli R, Wang J, Fox M. Contraception counseling, pregnancy intention and contraception use in women with medical problems: an analysis of data from the Maryland Pregnancy Risk Assessment Monitoring System (PRAMS). Contraception. 2013;88(2):263-268.

6. American College of Obstetricians and Gynecologists. ACOG Practice Bulletin No. 121: Long-acting reversible contraception: implants and intrauterine devices. Practice Bulletin No. 121. American College of Obstetricians and Gynecologists. Obstet Gynecol. 2011;118(1):184196.

7. Palinkas LA, Horwitz SM, Green CA, Wisdom JP, Duan N, Hoagwood K. Purposeful sampling for qualitative data collection and analysis in mixed method implementation research. Adm Policy Ment Health. 2015;42(5):533-544.

8. Atkins L, Francis J, Islam R, et al. A guide to using the Theoretical Domains Framework of behaviour change to investigate implementation problems. Implement Sci. 2017;12(1):7795.

9. Akers AY, Gold MA, Borrero S, Santucci A, Schwarz EB. Providers' perspectives on challenges to contraceptive counseling in primary care settings. J Womens Health (Larchmt). 2010;19(6):1163-1170.

10. Dehlendorf C, Levy K, Ruskin R, Steinauer J. Health care providers' knowledge about contraceptive evidence: a barrier to quality family planning care? Contraception. 2010;81(4):292298. 
11. Wu JP, Gundersen DA, Pickle S. Are the contraceptive recommendations of family medicine educators evidence-based? A CERA Survey. Fam Med. 2016;48(5):345-352.

12. Crabtree BF, Miller WL. Using codes and code manuals. a template organizing style of interpretation. In: Crabtree BF, Miller WL, eds. Doing qualitative research. 2nd ed. Thousand Oaks, CA: Sage; 1999:163-178.

13. Miles MB, Huberman AM. Early steps in analysis: Codes and coding. In: Miles MB, Huberman AM, eds. Qualitative data analysis:An expanded sourcebook. Second ed. Thousand Oaks, CA: Sage publications; 1994. 55-63.

14. Nelson J. Using conceptual depth criteria: addressing the challenge of reaching saturation in qualitative research. Qual Res. 2017;17(5):554-570.

15. Onwuegbuzie AJ, Leech NL. Validity and qualitative research: an oxymoron? Qual Quant. 2007;41(2):233-249.
16. Centers for Disease Control and Prevention United States Medical Eligibility Criteria (US MEC) for Contraceptive Use, 2016. 2016; https://www.cdc.gov/reproductivehealth/contraception/mmwr/mec/summary.html. Accessed October 8, 2018.

17. Rubin SE, Fletcher J, Stein T, Segall-Gutierrez P, Gold M. Determinants of intrauterine contraception provision among US family physicians: a national survey of knowledge, attitudes and practice. Contraception. 2011;83(5):42-478.

18. Thompson KM, Rocca CH, Stern L, et al. Training contraceptive providers to offer intrauterine devices and implants in contraceptive care: a cluster randomized trial. Am J Obstet Gynecol. 2018;218(6):597. e591-e597.

19. Rubin SE, Maldonado L, Fox K, Rosenberg R, Wall J, Prine L. Establishing and conducting a regional, hands-on long-acting reversible contraception training center in primary care Womens Health Issues. 2018;28(5):375-378.
20. Schwarz EB, Parisi SM, Handler SM, Koren G, Shevchik G, Fischer GS. Counseling about medication-induced birth defects with clinical decision support in primary care. J Womens Health (Larchmt). 2013;22(10):817-824.

21. Gomez AM, Fuentes L, Allina A. Women or LARC first? Reproductive autonomy and the promotion of long-acting reversible contraceptive methods. Perspect Sex Reprod Health. 2014;46(3):171-175.

22. Dehlendorf C, Grumbach K, Schmittdiel JA, Steinauer J. Shared decision making in contraceptive counseling. Contraception. 2017;95(5):452-455.

23. Russo JA, Chen BA, Creinin MD. Primary care physician familiarity with US medical eligibility for contraceptive use. Fam Med. 2015;47(1):15-21. 